\title{
Propagación mediante semilla botánica de Parastrephia quadrangularis y Baccharis tricuneata en San José de Aymará - Huancavelica, Perú
}

\author{
Propagation using seeds of Parastrephia quadrangularis and Baccharis tricuneata \\ in San Jose de Aymara - Huancavelica, Peru
}

\author{
Solange Montero Terry ${ }^{1, *}$
}

\begin{abstract}
Recibido: 15 setiembre 2020|Aceptado: 10 noviembre 2021|Publicado en línea: 29 diciembre 2021 Citación: Montero Terry, S. 2021. Propagación mediante semilla botánica de Parastrephia quadrangularis y Baccharis tricuneata en San José de Aymará - Huancavelica, Perú. Revista Forestal del Perú 36(2): 197-210. DOI: http://dx.doi.org/10.21704/rfp.v36i2.1798
\end{abstract}

\begin{abstract}
Resumen
En este estudio se presentan los resultados sobre la propagación por semillas en el vivero de la comunidad San José de Aymará durante el 2005. Se desea contribuir a mejorar la metodología de propagación de Parastrephia quadrangularis (Meyen) Cabrera y Baccharis tricuneata (Lam) Pers., ya que no hay mayores antecedentes para su manejo en el vivero. Se probaron tres tratamientos o técnica de propagación (bolsas de $10 \times 18 \mathrm{~cm}$, bolsas de $13 \times 18 \mathrm{~cm}$ y platabanda) y tres sustratos: 1) turba: materia orgánica: tierra agrícola, 2) arena: materia orgánica: tierra agrícola, y 3) materia orgánica: tierra agrícola, obteniendo un total de 9 combinaciones. Considerándose en cada combinación los siguientes parámetros: supervivencia en el vivero, altura $(\mathrm{cm})$ y perímetro al cuello de raíz $(\mathrm{cm})$. Se obtuvo que ambas especies responden favorablemente a la propagación utilizando semillas. Resultando para $P$. quadrangularis la mejor combinación bolsas de $13 \times 18 \mathrm{~cm}$ y sustrato (materia orgánica: tierra agrícola), obteniendo un incremento promedio en altura entre 0.71 - $0.91 \mathrm{~cm} / \mathrm{mes}$; esta especie llega a desarrollarse en todos los sustratos empleados en el vivero. Por otro lado, B. tricuneata obtuvo mejores resultados utilizando bolsas $13 \times 18 \mathrm{~cm}$ y sustrato (materia orgánica: tierra agrícola: turba), obteniendo un incremento promedio en altura entre $1.04-1.09$ $\mathrm{cm} / \mathrm{mes}$. Sin embargo, resultó nulo el uso del sustrato (materia orgánica: tierra agrícola: arena). La alta mortalidad inicial en la plantación indica que todas las plantas desarrolladas en platabandas (a raíz desnuda), para ambas especies, requieren de más tiempo en el vivero e investigación.
\end{abstract}

Palabras clave: arbustos, tolas, propagación por semilla, puna, sustratos

\footnotetext{
${ }^{1}$ Facultad de Ciencias Forestales, Universidad Nacional Agraria La Molina (UNALM), Av. La Molina s/n, La Molina, Lima, Perú.

*Autor de Correspondencia: solange.mt@gmail.com
} 


\begin{abstract}
This study presents the results of seed propagation at the nursery of San José de Aymará community in 2005. The study aimed to contribute to improve the propagation methodology of Parastrephia quadrangularis (Meyen) Cabrera and Baccharis tricuneata (Lam) Pers., since there is no greater background for their management at nursery. Therefore, three treatments (bags of $10 \times 18 \mathrm{~cm}$, bags of $13 \times 18 \mathrm{~cm}$ and bareroot) and three soil mix were tested: 1) turban: organic matter: agricultural land, 2) sand: organic matter: agricultural land, and 3) organic matter: agricultural land, obtaining a total of 9 combinations. The following parameters are considered in each combination: survival in the nursery, height $(\mathrm{cm})$ and perimeter at the root neck $(\mathrm{cm})$. It was obtained that both species respond favourably to propagation using seeds. Resulting for P. quadrangularis the best combination bags of $13 \times 18 \mathrm{~cm}$ and soil mix (organic matter: agricultural land), obtaining an average increase in height between $0.71-0.91 \mathrm{~cm} / \mathrm{month}$; this species develops on all soil mixes used in the nursery. On other hand, B. tricuneata achieved better results using bags $13 \times 18 \mathrm{~cm}$ and soil mix (organic matter: agricultural land: peat), obtaining an average increase in height between $1.04-1.09 \mathrm{~cm} /$ month. However, the use of the soil mix (organic matter: agricultural land: sand) proved null and void. In addition, the high mortality (in early establishment) for both species indicates that all plants developed using treatment (bareroot) could require more time in the nursery and more research.
\end{abstract}

Key words: shrubs (bushes), tolas, propagation by seeds, puna, soil mix

\section{Introducción}

En muchas de las zonas altoandinas de nuestro país el uso de leña como fuente de energía ha generado una gran disminución de gran parte del componente vegetal leñoso. En comunidades que se localizan sobre los $3800 \mathrm{~m}$ la satisfacción de la necesidad de leña como combustible puede llevar rápidamente al deterioro de los recursos forestales existentes; y cuando estos se agotan se utilizan los residuos agrícolas y pecuarios como la bosta (estiércol seco de vaca) (Reynel 1988). Además, el deterioro de los recursos forestales incrementa el riesgo ocasionado por un aumento de la escorrentía durante la estación de lluvia, lo cual podría ocasionar erosión de los suelos. Según Turner et al. (2003), la vulnerabilidad de los ecosistemas, por ejemplo, en el ecosistema andino, proviene como resultado de "riesgos", los cuales pueden ser de dos tipos: riesgo ocasionado por perturbaciones extremas, y, riesgos normalmente asociados a la actividad de los seres humanos, como cuando se usa leña y bosta para cocinar. El uso intenso de bosta puede causar una reducción del potencial agrícola y la posibilidad de producción de alimentos. En la comunidad
San José de Aymará (SJA) el uso de bosta ocurre y se utiliza mezclada con leña normalmente de Polylepis sp. y Eucalyptus sp., siendo esta última la leña para cocinar de uso más común ${ }^{1}$. Por lo tanto, el manejo de arbustos en la comunidad podría ser una buena alternativa frente a la depredación de árboles y bosta.

En la región andina existen especies arbustivas que son reconocidas por las comunidades tradicionales como excelente combustible para leña, estas especies se encuentran en especial en zonas del sur y centro del país: Arequipa, Ayacucho, Lima, Puno y Tacna (Cabrera 1978, Brako y Zarucchi 1993, Martínez 2002); formando la comunidad ecológica tipo tolar (Weberbauer 1945). El tolar (estepa arbustiva) es una comunidad de arbustos perennes y siempre verdes de terreno árido (Weberbauer 1945, Cabrera 1978). Y de acuerdo con las ecorregiones de Brack Egg (1986), estas se encuentran en la puna, y en el piso alto de la serranía esteparia. En esta ecorregión, el poder calórico de la especie dominante del tolar (Parastrephia spp.) es de gran utilidad y es empleado como combustible vegetal en la estación de lluvias, debido a que prende aun cuando se encuentra

\footnotetext{
${ }^{1}$ Comunero de SJA. 2004. Comunicación oral. San José de Aymará, Huancavelica, Perú.
} 
"verde" (Cabrera 1978). Asimismo, Baccharis tricuenata (especie también presente en el tolar) es de gran utilidad en prácticas vinculadas con la conservación de suelos debido a un sistema radicular bien desarrollado y su rusticidad (Reynel y Felipe-Morales 1987). En Puno, es utilizada en el manejo agroforestal tradicional y es componente común de cercos vivos (Reynel y Felipe-Morales 1987, Borel 1990). Ambos arbustos pueden ser encontrados entre los 2000 - 4500 m (Brako y Zaruchi 1993).

Estudios preliminares de propagación por semillas dieron buenos resultados para especies del género Parastrephia (Reynel 1988); y pobladores de Puno indican que la propagación por semilla de $B$. tricuneata es exitosa. Sin embargo, en esos estudios no se encontraron datos que cuantifiquen el manejo en el vivero, los tratamientos empleados, dimensiones de las plantas ni el porcentaje de supervivencias una vez instaladas en campo definitivo.

Por lo descrito, los objetivos del estudio de propagación fueron: 1. evaluar tres tratamientos de propagación para $P$. quadrangularis y B. tricuneata (el porcentaje de mortalidad), 2. evaluar tres sustratos de propagación para ambas especies, y 3. cuantificar el crecimiento mensual en altura de ambas especies bajo condiciones de vivero. Además, para complementar los resultados en el vivero y determinar la influencia de los tratamientos en campo, se evaluó la supervivencia (inicial) en plantación en las tierras de la comunidad un mes después de ser instaladas.

\section{Materiales y Métodos}

\section{Área de estudio}

La comunidad de SJA se localiza en el distrito de Pazos, provincia de Tayacaja, en el departamento de Huancavelica a una altura de $3900 \mathrm{~m}$; con una temperatura media entre 9.45 - $10.95^{\circ} \mathrm{C}$, con precipitación estacional entre 85.7 y $90.2 \mathrm{~mm}$ (parámetros climáticos de la estación Acostambo a 3650 m correspondientes al año 2005; ver Cuadro 1). La plantación se realizó a una altura de 3924 m en las tierras de la comunidad.

\section{Análisis estadístico (vivero)}

Debido a la naturaleza del estudio en el que se observa simultáneamente dos factores se usó el diseño factorial, con distribución al azar de las unidades experimentales. Para lo cual, se trabajó con cada especie dos factores A y B, con tres tratamientos cada uno, el número de combinaciones fue de 9 con 3 repeticiones, lo que representa un total de 27 unidades experimentales por especie, las cuales estuvieron conformadas por grupos de 23 plantas. Los factores y claves (ambos tamaños de bolsa en dimensión plana):

\section{FACTOR A: Técnica de propagación}

$$
\begin{gathered}
\text { a1 - Bolsa de } 10 \times 18 \mathrm{~cm} \\
\text { a2 - Bolsa de } 13 \times 18 \mathrm{~cm} \\
\text { a3 - Platabanda }
\end{gathered}
$$

\section{FACTOR B: Tipo de sustrato}

b1 - Turba + Materia orgánica + Tierra agrícola

b2 - Arena + Materia orgánica + Tierra agrícola

b3 - Materia orgánica + Tierra agrícola

Con cada factor se creó las "claves" de las 9 combinaciones, por ejemplo, alb1 (Bolsa de 10 $\times 18 \mathrm{~cm}$ y sustrato Turba + Materia orgánica + Tierra agrícola). Lo que resulta en un total de 621 plantas por especie. El modelo aditivo lineal del estudio fue: $\mathrm{Y}_{\mathrm{ijk}}=\mu+\alpha_{\mathrm{i}}+\beta_{\mathrm{j}}+(\alpha \beta)_{\mathrm{ij}}+$ $\mathrm{e}_{\mathrm{ijk}}$. Donde:

$\mathrm{Y}_{\mathrm{ij \textrm {j }}}$ : respuesta observada (altura, perímetro y supervivencia)

$\mu$ : parámetro del modelo estimado por la media de $\mathrm{Y}$

$\alpha_{i}$ : efecto de la técnica de propagación

$\beta_{\mathrm{j}}$ : efecto del sustrato

$(\alpha \beta)_{\mathrm{ij}}$ : efecto de la interacción

$e_{i \mathrm{ij}}:$ error experimental en la unidad observada

\section{Análisis estadístico (plantación)}

El análisis del diseño no incluyó la influencia de los sustratos ya que este factor fue analizado en el vivero. El diseño empleado fue de bloques 


\begin{tabular}{|c|c|c|c|c|c|c|c|c|c|c|c|}
\hline \multicolumn{12}{|c|}{$\mathbf{T}^{\mathbf{0}} \max \left({ }^{\circ} \mathbf{C}\right)$} \\
\hline Año & 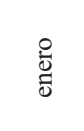 & 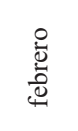 & $\begin{array}{l}\stackrel{\text { N }}{\mathbb{\Xi}} \\
\text { J }\end{array}$ & $\begin{array}{l}\overline{\bar{\sigma}} \\
\text { त् }\end{array}$ & 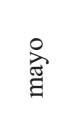 & 亶 & $\stackrel{\varrho}{\Xi}$ & 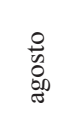 & 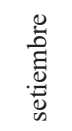 & $\begin{array}{l}0 \\
\text { : } \\
0 \\
0\end{array}$ & 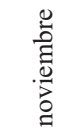 \\
\hline 2005 & 17.8 & 17.1 & 17 & 17.3 & 18.5 & 18 & 17.8 & 18.1 & 17.9 & 17.2 & 18.4 \\
\hline \multicolumn{12}{|c|}{$\mathrm{T}^{\mathbf{0}} \min \left({ }^{\circ} \mathrm{C}\right)$} \\
\hline Año & 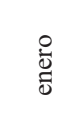 & 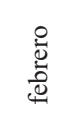 & $\begin{array}{l}\text { N } \\
\text { : } \\
\text { Z }\end{array}$ & $\begin{array}{l}\bar{E} \\
\text { त् }\end{array}$ & 胥 & 音 & $\stackrel{\varrho}{\Xi}$ & $\begin{array}{l}0 \\
\stackrel{0}{0} \\
\stackrel{0}{0} \\
\text { d] }\end{array}$ & 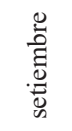 & 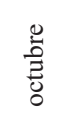 & 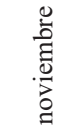 \\
\hline 2005 & 4.1 & 4.5 & 4.8 & 3 & 1.5 & 2.1 & 1.4 & 0.8 & 2.6 & 3.8 & 3.1 \\
\hline \multicolumn{12}{|c|}{$\mathrm{T}^{\circ}$ media $\left({ }^{\circ} \mathrm{C}\right)$} \\
\hline Año & 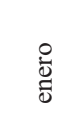 & 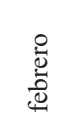 & $\begin{array}{l}\stackrel{\stackrel{N}{\sharp}}{\overparen{\Xi}} \\
\text { }\end{array}$ & $\begin{array}{l}\bar{E} \\
\text { है }\end{array}$ & 蔦 & 具 & $\stackrel{\ominus}{\Xi}$ & 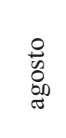 & 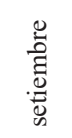 & 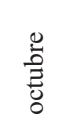 & 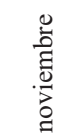 \\
\hline 2005 & 10.95 & 10.8 & 10.9 & 10.15 & 10 & 10.05 & 9.6 & 9.45 & 10.25 & 10.5 & 10.75 \\
\hline \multicolumn{12}{|c|}{$\mathbf{P P}(\mathbf{m m})$} \\
\hline Año & $\begin{array}{l}\stackrel{\circ}{\mathscr{D}} \\
\stackrel{0}{0}\end{array}$ & 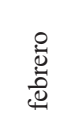 & $\begin{array}{l}\text { N } \\
\text { ज्ञ } \\
\text { I }\end{array}$ & $\begin{array}{l}\overline{\mathrm{E}} \\
\bar{\sigma}\end{array}$ & 胥 & 息 & $\stackrel{\varrho}{\Xi}$ & 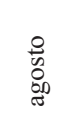 & 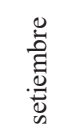 & 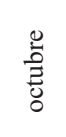 & 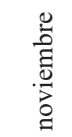 \\
\hline 2005 & 68.5 & 100.8 & 85.7 & 41.2 & 19.4 & 0 & 6 & 4.5 & 24.5 & 90.2 & 56 \\
\hline
\end{tabular}

Cuadro 1. Parámetros climáticos del periodo enero - noviembre 2005 de la estación Acostambo a 3650 m. Fuente: Tomado de SENAMHI (2005).

completos al azar (DBCA) con tres repeticiones y nueve plantas por unidad experimental (parcelas), y tres bloques por especie. Con nueve parcelas por bloque - 243 plantas para $P$. quadrangularis y seis parcelas por bloque - 162 plantas para $B$. tricuenata.

\section{Desarrollo del estudio}

El estudio se desarrolló en cuatro etapas, de las cuales dos (etapa 3 y 4) fueron realizadas en la comunidad SJA. A continuación, el proceso del estudio por etapa en orden cronológico:

Etapa 1: se realizó la colecta de las semillas, con las cuales se realizó los ensayos de semillas.

Etapa 2: propagación mediante semilla en camas de almacigo hasta que estuvieron listas para el repique.
Etapa 3: repique, trasplante y monitoreo del desarrollo en el vivero de la comunidad

Etapa 4: se realizó la plantación y evaluación de sobrevivencia de los arbustos de tola en las tierras de la comunidad.

\section{Etapa 1: Recolección de semillas}

Las semillas se colectaron a mediados del mes de septiembre del 2004, época previa a la temporada de lluvias, se determinó esta época en relación con lo observado según Pérez Mercado (1994). Se confirmó el estado fenológico de los arbustos con personas cercanas al área de los tolares en el departamento de Arequipa, en la provincia de Caylloma.

Se consideró semillas maduras cuando al mover la rama donde se encontraba estas se 


\begin{tabular}{|c|c|c|c|c|c|c|}
\hline Sustratos & pH (1:1) & M.O. \% & Arena \% & Limo \% & Arcilla \% & Clase textural \\
\hline b1 & 6.4 & 3.3 & 76 & 20 & 4 & Arena franca \\
\hline b2 & 5.9 & 2.9 & 76 & 22 & 2 & Arena franca \\
\hline b3 & 3.8 & 9.2 & 44 & 42 & 14 & Franco \\
\hline
\end{tabular}

Cuadro 2. Análisis mecánico, clase textural, materia orgánica y pH de sustratos.

caían con facilidad y eran de un color pardo oscuro, sin llegar a ser negro, nunca verde.

Para la colecta se utilizó bolsas de cierre hermético (para frutos y semillas), tijeras de podar, etiquetas para poder identificar muestras, y bolsas (sacos) para muestras botánicas de los arbustos. Las muestras botánicas fueron preparadas y secadas el mismo día de colecta para evitar daños en las muestras; las muestras se colectaron para corroborar en el herbario la identificación en campo de las especies.

Ensayos de semillas: Con las semillas colectadas se realizaron los siguientes ensayos: a) análisis de pureza, para determinar la composición del lote de semillas y la cantidad de impurezas (otras semillas y materia inerte) constituyentes de la muestra de semillas recolectadas en Arequipa; y b) prueba de germinación, para lo cual se utilizó placas de Petri, papel filtro y fungicida en polvo (Benlate). En cada placa se colocó 50 semillas puras, teniendo un total de 400 semillas por especie, las cuales se observaron diariamente hasta culminar la germinación. Se empleo las siguientes condiciones de germinación: Temperatura $8-22{ }^{\circ} \mathrm{C}$, y fotoperiodo de 8 horas.

\section{Etapa 2: Preparación de la cama de Almácigo}

La profundidad de las camas de almácigo fue de $12 \mathrm{~cm}$ y medidas de $10 \times 1 \mathrm{~m}$, se utilizó un sustrato único para ambas especies; arena de cantera: materia orgánica: musgo (3:2:1).

El sustrato fue desinfectado con bromuro de metilo, utilizando una concentración como la sugerida en PRONAMACHCS (1998). Las camas se regaron 24 horas previas a la siembra a razón de cuatro litros de agua por $\mathrm{m}^{2}$. Las semillas no recibieron tratamiento alguno previo a la siembra en el almacigo.
El método de siembra fue al voleo y se cubrió las semillas con una capa fina de sustrato y paja, y se regó a razón de un litro de agua por $\mathrm{m}^{2}$ con regadera de lluvia. Se almacigó 27.8 gr de semilla pura de $B$. tricuneata en un área de $8.6 \mathrm{~m}^{2}$, y 13.3 gr de semilla pura de $P$. quadrangularis en $4.85 \mathrm{~m}^{2}$. Para proteger y evitar pérdidas de las semillas por exposición al viento y los rayos del sol, se cubrió con malla de sombra típica de viveros y durante días de lluvia se procuró cubrir la cama de almácigo con plástico transparente, el cual se retiraba cuando la lluvia terminaba. El único tratamiento cultural que se brindó consistió en el deshierbe de la almaciguera.

\section{Etapa 3: Repique}

La almaciguera fue regada 24 horas previas al repique. Para la selección de plantas se siguió el siguiente criterio: el número de hojas verdaderas (con 4 - 6 hojas), apariencia, presencia de raíces secundarias y que la raíz principal no esté deforme sino recta. Esto se llevó a cabo hasta completar 621 plantas para las 27 unidades de muestra tratamiento/sustrato/especie (se consideró un margen de pérdida de 5\%). Inmediatamente después de seleccionar las plantas, estas fueron transportadas a la comunidad de San José de Aymará. Para transportarlas se utilizó bandejas de $0.30 \times 0.20 \mathrm{~m}$ con sustrato húmedo, las plantas fueron agrupadas en ramilletes de 23 plantas y para la desinfección de las raíces se aplicó un fungicida (Benlate), la concentración del fungicida siguió la guía de PRONAMACHCS (1998).

En esta etapa se monitoreo el incremento mensual $(\mathrm{cm})$ y altura aérea $(\mathrm{cm})$; se entiende por altura aérea la sección desde el cuello de raíz hasta el final del tallo principal (PRONAMACHCS 1998). Una vez culminado el desarrollo de las plantas en las camas de repique, se cuantificó el perímetro al cuello de 
raíz $(\mathrm{cm})$ de todas las plantas vivas en cada uno de los tratamientos; se define perímetro al cuello de raíz, a la longitud del contorno del tallo ubicado en la base de la planta entre $1-2 \mathrm{~cm}$ antes de iniciarse la raíz (PRONAMACHCS 1998). Esta etapa demando labores culturales de: deshierbe, poda de raíces para las plantas en platabandas al octavo mes y a los diez días antes de la plantación, y manejo de tinglado al inicio del repique y en época de heladas (ver Figuras 1 y 2). El análisis mecánico, clase textural, materia orgánica y $\mathrm{pH}$ de los sustratos se realizó para apoyar al análisis de los resultados (ver Cuadro 2).

\section{Etapa 4: Plantación}

Previo a la plantación se realizó la buena práctica de riego copioso ( 24 horas antes) en el vivero, a razón de cinco litros de agua por $\mathrm{m}^{2}$ aproximadamente. El área de las parcelas o unidades experimentales de la plantación fue de $4 \mathrm{~m}^{2}$ y el distanciamiento entre plantas fue de un metro. La evaluación del porcentaje de supervivencia se realizó en diciembre del 2005, donde se contabilizó el total de las plantas vivas en cada unidad experimental para determinar la influencia de los tratamientos.

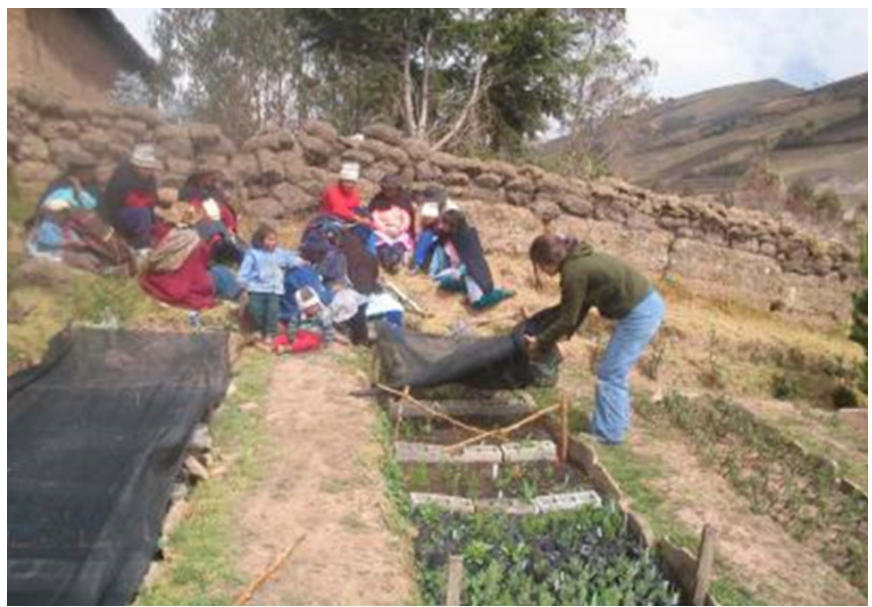

Figura 1. Manejo del tinglado en el vivero de la comunidad San José de Aymará - Huancavelica, Perú.

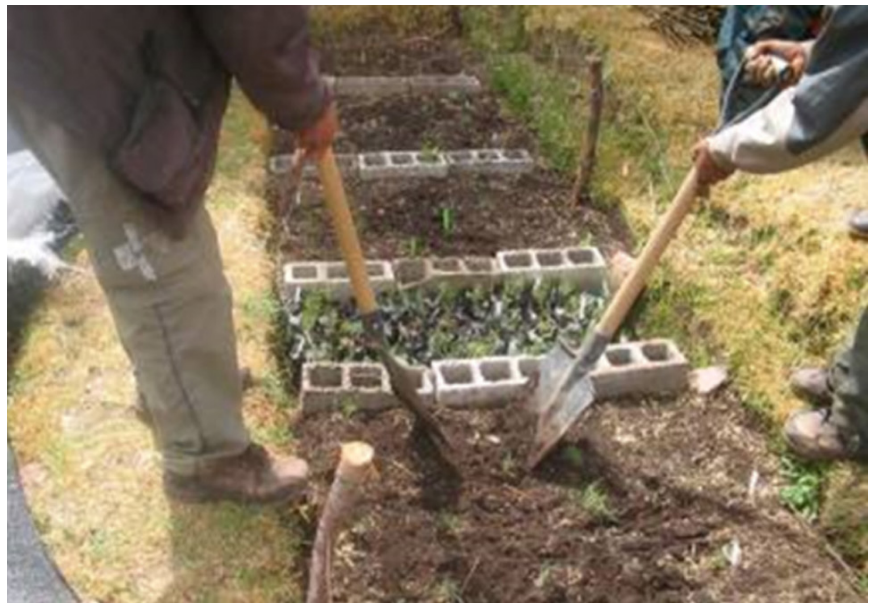

Figura 2. Manejo de platabanda: poda de raíz de la comunidad San José de Aymará - Huancavelica, Perú. 


\section{Resultados}

\section{Etapa 1: Ensayos de semillas}

En el análisis de pureza, se encontró mucho material inerte con las semillas 0.3008 gramos en 500 semillas de $B$. tricuneata, y 0.5835 gramos en 1200 semillas de $P$. quadrangularis esto se debe posiblemente a las características de las semillas, son muy volátiles y captan con facilidad humedad y polvo (ver Cuadro 3).

La prueba de germinación mostró que las semillas de $B$. tricuneata y $P$. quadrangularis tenían un incremento de germinación estable. Entre el octavo y el decimosexto día es donde la germinación de las semillas ocurrió para ambas especies, sin embargo, la prueba de germinación duro un total de 44 días, con un 26\% de germinación al final del periodo de prueba para B. tricuneata; y un total de 35 días con un $46 \%$ para $P$. quadrangularis (ver Figuras 3 y 4 ).

\section{Etapa 2: Almácigo}

La emergencia total de las semillas en la cama de almácigo duró de 25 a 32 días para ambas especies. Parastrephia quadrangularis permaneció en el almácigo 105 días, estando lista para el repique con una altura promedio de $2.9 \mathrm{~cm}$ y en promedio con 9 hojas verdaderas.

Las plantas de B. tricuneata permanecieron 29 días más en las camas de almácigo, ya que su desarrollo a los 105 días no era suficiente para ejecutar el repique; se consideró lista para el repique cuando alcanzaron una altura promedio de $3 \mathrm{~cm}$ y con más de 4 hojas verdaderas.

\section{Etapa 3: Repique}

El repique duro un total de 281 días para $P$. quadrangularis y 252 días para B. tricuenata. Para P. quadrangularis el número total de plantas vivas fue de 450 plantas que representa el $72.5 \%$ de lo que se trasplantó (ver Cuadro 4). Estas permanecieron en el vivero nueve meses (marzo - noviembre).

En el caso de B. tricuneata se analizaron los efectos de solo dos sustratos, visto que todas las plantas que se trabajaron en el sustrato b2 (sustrato arenoso) se perdieron (ver Cuadro 5). El número total de plantas vivas al final del repique empleado sustrato b1 y b3 fue de 336 plantas que representa el $54 \%$ de lo que se sembró. Estas permanecieron en el vivero ocho meses (abril - noviembre).

Alturas (cm): Para B. tricuneata todas las combinaciones que emplearon sustrato b1 (sustrato con turba) resultaron con un desarrollo mayor en altura promedio en comparación con el sustrato b3. Con incrementos promedio de $1.04 \mathrm{~cm}$ y $0.9 \mathrm{~cm}$ empleado b1 y b3 respectivamente (ver Cuadro 6). Para P. quadrangularis no existe diferencias significativas cuando se trabajaron las plantas en ambos tamaños de bolsas. Con incrementos promedio de $0.64 \mathrm{~cm}$, $0.92 \mathrm{~cm}$ y $0.97 \mathrm{~cm}$ empleando los sustratos b2, b1 y b3 respectivamente (ver Cuadro 6).

Perímetro al cuello de raíz: Para B. tricuneata el análisis de variancia divide los resultados en dos grupos, mostrando que existe mayor desarrollo del perímetro al cuello de raíz en plan-

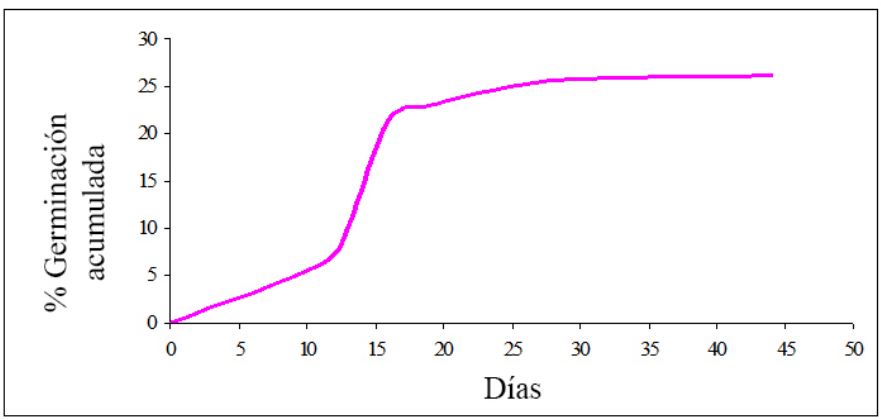

Figura 3. Curva de germinación de B. tricuneata en condiciones de laboratorio. 


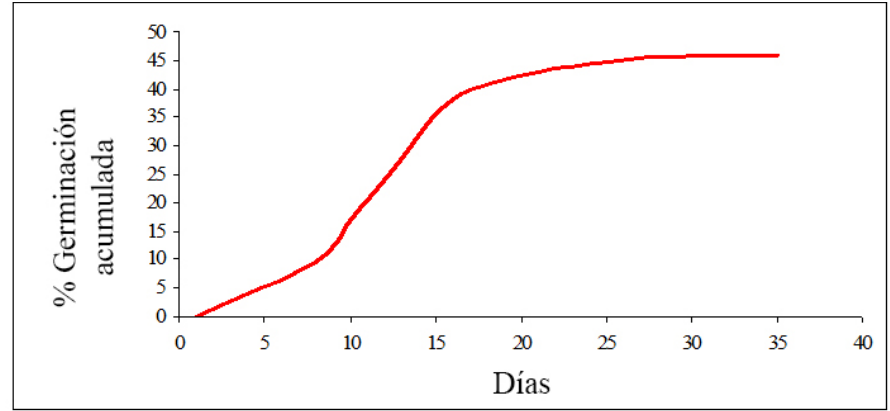

Figura 4. Curva de germinación de P. quadrangularis en condiciones de laboratorio.

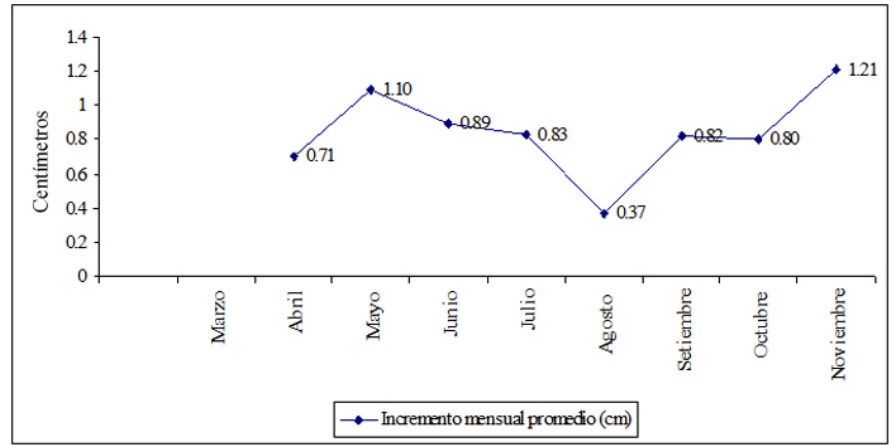

Figura 5. Incremento mensual de altura promedio de P. quadrangularis.

tas trabajadas en platabanda con sustratos bl (sustrato con turba) en comparación con plantas trabajadas en las otras combinaciones (ver Cuadro 6). Para P. quadrangularis los resultados indican que no existe significancia entre los factores, y se reportó el coeficiente de variabilidad de $16.30 \%$ y un promedio de perímetro al cuello de raíz $1.39 \mathrm{~cm}$ (ver Cuadro 6).

Crecimiento Mensual: Para P. quadrangularis se obtuvo un incremento promedio de $0.64 \mathrm{~cm}$ cuando se trabajó con el sustrato arenoso b2, mientras que con el sustrato b3 se obtuvo un incremento de $0.97 \mathrm{~cm}$ (ver Cuadro 6 y Figura 5). Para B. tricuneata se obtuvo un incremento promedio de $1.04 \mathrm{~cm}$ empleando sustrato b1 (con turba), y un incremento de $1.09 \mathrm{~cm}$ empleando la técnica a2 bolsas de $13 \times 18 \mathrm{~cm}$ (ver Cuadro 6 y Figura 6).

\section{Etapa 4: Supervivencia en campo definitivo}

La altura promedio cuando se llevaron a campo definitivo para $P$. quadrangularis fue de
$8.86 \mathrm{~cm}$, y para B. tricuneata fue de $10.67 \mathrm{~cm}$. $\mathrm{Al}$ mes de instalada la plantación se reportó un alto porcentaje de supervivencia. Para $P$. quadrangularis fue de 95\% para las plantas desarrolladas con tratamientos a 2 y a 1 (ambos tamaños de bolsa). Sin embargo, solo se encontró $27 \%$ de las plantas desarrolladas en platabandas. Para B. tricuneata las plantas desarrolladas en bolsas, tratamientos a1 y a2 mostraron $94 \%$ y $100 \%$ de supervivencia respectivamente. No obstante, se perdió gran cantidad de plantas desarrolladas en platabandas quedando solo el $31 \%$ de lo que se plantó.

\section{Discusión}

\section{Etapa 1: Ensayo de semillas}

El porcentaje acumulado de germinación fue de $46 \%$ para $P$. quadrangularis y $26 \%$ para $B$. tricuneata; lo que confirmo lo que otros estudios de arbustos de tola sugerían, que las tolas se pueden propagar fácilmente utilizando se- 


\begin{tabular}{|c|c|c|c|c|c|}
\hline Especie & $\begin{array}{c}\text { Cantidad } \\
\text { (número de } \\
\text { semillas) }\end{array}$ & $\begin{array}{c}\text { Semillas } \\
\text { Puras (gr) }\end{array}$ & $\begin{array}{c}\text { Material } \\
\text { Inerte (gr) }\end{array}$ & Impureza \% & Pureza \% \\
\hline Baccharis tricuneata & 500 & 0.2142 & 0.3008 & 58 & 42 \\
\hline $\begin{array}{c}\text { Parastrephia } \\
\text { quadrangularis }\end{array}$ & 1200 & 0.5452 & 0.5835 & 52 & 48 \\
\hline
\end{tabular}

Cuadro 3. Resultados de purezas y de impurezas de las semillas B. tricuenata y P. quadrangularis.

\begin{tabular}{|c|c|c|c|c|c|c|c|c|c|}
\hline \multirow{2}{*}{ Factor } & \multicolumn{3}{|c|}{ a1 } & \multicolumn{3}{|c|}{$\mathbf{a 2}$} & \multicolumn{3}{|c|}{$\mathbf{a 3}$} \\
\hline & \multirow[t]{2}{*}{ b1 } & \multirow[t]{2}{*}{ b2 } & \multirow{2}{*}{ b3 } & \multirow{2}{*}{ b1 } & \multirow[t]{2}{*}{ b2 } & \multirow{2}{*}{ b3 } & \multirow[t]{2}{*}{ b1 } & \multirow[t]{2}{*}{ b2 } & \multirow[t]{2}{*}{ b3 } \\
\hline Repetición & & & & & & & & & \\
\hline 1 & $78 \%$ & $78 \%$ & $70 \%$ & $91 \%$ & $78 \%$ & $96 \%$ & $48 \%$ & $70 \%$ & $39 \%$ \\
\hline 2 & $96 \%$ & $48 \%$ & $83 \%$ & $96 \%$ & $57 \%$ & $100 \%$ & $70 \%$ & $70 \%$ & $78 \%$ \\
\hline 3 & $78 \%$ & $52 \%$ & $87 \%$ & $83 \%$ & $74 \%$ & $83 \%$ & $70 \%$ & $57 \%$ & $30 \%$ \\
\hline \multirow[t]{2}{*}{ Promedio Combinado ab } & $84 \%$ & $59 \%$ & $80 \%$ & $90 \%$ & $70 \%$ & $93 \%$ & $62 \%$ & $65 \%$ & $49 \%$ \\
\hline & \multicolumn{3}{|c|}{1} & \multicolumn{3}{|c|}{2} & \multicolumn{3}{|c|}{3} \\
\hline Media de a & \multicolumn{3}{|c|}{$74 \%$} & \multicolumn{3}{|c|}{$84 \%$} & \multicolumn{3}{|c|}{$59 \%$} \\
\hline Media de $b$ & \multicolumn{3}{|c|}{$79 \%$} & \multicolumn{3}{|c|}{$65 \%$} & \multicolumn{3}{|c|}{$74 \%$} \\
\hline $\begin{array}{c}\text { Promedio total } \\
\text { Supervivencia \% }\end{array}$ & \multicolumn{9}{|c|}{72.5} \\
\hline Coeff. Variabilidad & \multicolumn{9}{|c|}{10.02} \\
\hline
\end{tabular}

Cuadro 4. Porcentaje de supervivencia por combinación y coeficiente de variabilidad de los datos al final del repique P. quadrangularis.

\begin{tabular}{|c|c|c|c|c|c|c|}
\hline \multirow{3}{*}{$\begin{array}{c}\text { Factor } \\
\text { Repetición }\end{array}$} & \multicolumn{2}{|c|}{ a1 } & \multicolumn{2}{|c|}{$\mathbf{a 2}$} & \multicolumn{2}{|c|}{ a3 } \\
\hline & \multirow[t]{2}{*}{ b1 } & \multirow[t]{2}{*}{ b3 } & \multirow[t]{2}{*}{ b1 } & \multirow[t]{2}{*}{ b3 } & \multirow[t]{2}{*}{ b1 } & \multirow[t]{2}{*}{ b3 } \\
\hline & & & & & & \\
\hline 1 & $96 \%$ & $74 \%$ & $91 \%$ & $96 \%$ & $87 \%$ & $65 \%$ \\
\hline 2 & $78 \%$ & $96 \%$ & $78 \%$ & $78 \%$ & $83 \%$ & $70 \%$ \\
\hline 3 & $87 \%$ & $61 \%$ & $87 \%$ & $87 \%$ & $74 \%$ & $74 \%$ \\
\hline \multirow[t]{2}{*}{ Promedio Combinado ab } & $87 \%$ & $77 \%$ & $86 \%$ & $87 \%$ & $81 \%$ & $70 \%$ \\
\hline & \multicolumn{2}{|c|}{1} & \multicolumn{2}{|c|}{2} & \multicolumn{2}{|c|}{3} \\
\hline Media de a & \multicolumn{2}{|c|}{$82 \%$} & \multicolumn{2}{|c|}{$86 \%$} & \multicolumn{2}{|c|}{$75 \%$} \\
\hline Media de $b$ & \multicolumn{2}{|c|}{$85 \%$} & \multicolumn{2}{|c|}{-} & \multicolumn{2}{|c|}{$78 \%$} \\
\hline $\begin{array}{l}\text { Promedio total } \\
\text { Supervivencia \% }\end{array}$ & \multicolumn{6}{|c|}{81.16} \\
\hline Coeff. Variabilidad & \multicolumn{6}{|c|}{11.98} \\
\hline
\end{tabular}

Cuadro 5. Porcentaje de supervivencia por combinación y coeficiente de variabilidad de los datos al final del repique $B$. tricuneata. 
Propagación mediante semilla de Parastrephia quadrangularis

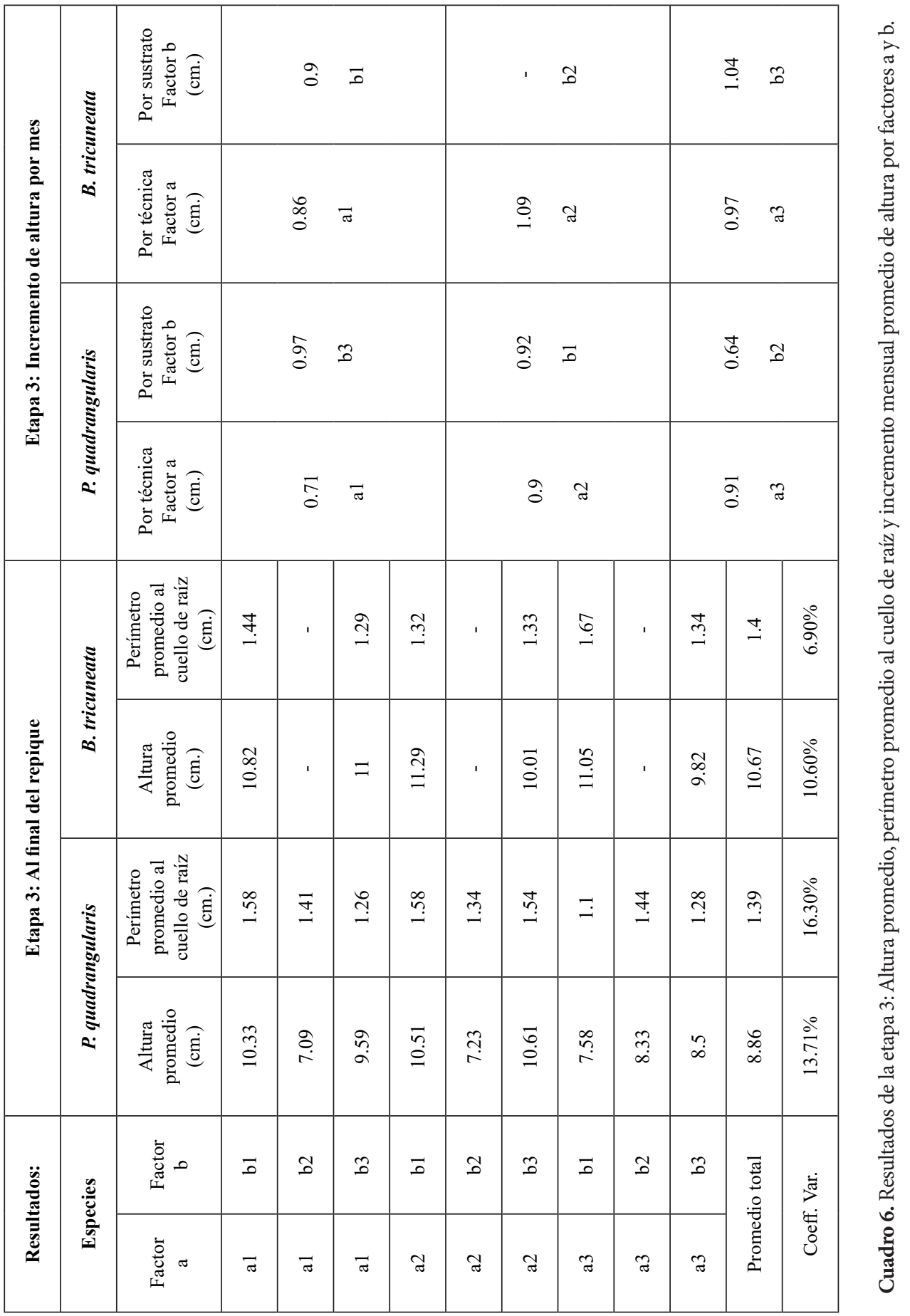




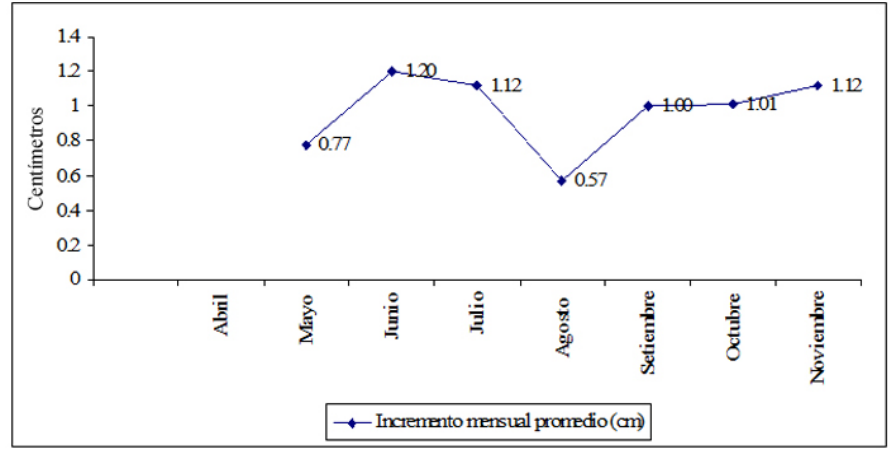

Figura 6. Incremento mensual de altura promedio de B. tricuneata.

millas de origen natural (Reynel 1988, Pérez Mercado 1994). Sin embargo, los picos de germinación entre el octavo y decimosexto día, y la continuación de la germinación hasta el día 35 para $P$. quadrangularis y 44 días para $B$. tricuneata se atribuyeron a que en el momento de colectar las semillas no todas habían alcanzado su maduración botánica y fisiológica. Ya que en otro estudio con especie del tolar, se menciona que los porcentajes de germinación son relativamente altos (Pérez Mercado 1994). Por ejemplo: ensayos de germinación con semillas de Parastrephia lepidhophylla Wedd., obtuvieron un porcentaje de germinación entre $76-90 \%$, este rango se atribuyó a las zonas donde se colectaron las semillas (Pérez Mercado 1994).

\section{Etapa 2: Almácigo}

Se observó que $B$. tricuneata requirió de un total de 134 días para alcanzar una altura promedio de $3 \mathrm{~cm}$, mientras $P$. quadrangularis a los 105 días alcanzó una altura promedio de $2.9 \mathrm{~cm}$ y nueve hojas verdaderas. Este mismo patrón se observó en los ensayos de semillas, lo cual se puede atribuir a la viabilidad (no todas las semillas habían alcanzado su maduración botánica y fisiológica); y el total de días en la cama de almacigo podría atribuirse a la variabilidad genética de la semilla botánica (Hartmann y Kester 1980). Ya que se sabe, que en condiciones de invernadero utilizando Parastrephia lepidhophylla, se obtuvo a los 30 días de almácigo plantas con 9 - 13 hojas y a los 60 días con 2 - 3 ramitas, alcanzando una altura de 3 - $4 \mathrm{~cm}$ (Pérez Mercado 1994). Por otro lado, para B. tricuneata no se encontró datos para poder comparar el tiempo en el almácigo, sin embargo, fue similar a resultados de estudios realizados en viveros en Puno, donde las plantas de tolas se repican a los 120 días y sé llevan a campo definitivo a los 180 días (Dueñas 2001, IIPSC QOLLASUYO 2001).

\section{Etapa 3: Repique}

A los diez meses del repique para P. quadrangularis, se pudo observar que la combinación a2b3, es la combinación que brinda mejores resultados con incremento de altura promedio de más de 0.9 cm y $93 \%$ de supervivencia; estos resultados se pueden atribuir al sustrato que tiene un alto contenido de materia orgánica y textura de suelo franco con $\mathrm{pH}$ 3.8. Sin embargo, se observó que trabajando con este mismo sustrato en platabandas (a3) se perdieron muchas plantas con solo $49 \%$ de ellas aptas para campo definitivo, esto posiblemente se debe a que se pierde más rápido la humedad en el suelo de la platabanda, por la mayor área expuesta a la superficie. Además, el resultado de la combinación a1b2 de 59\% también sugiere que la humedad en el sustrato influencia los porcentajes de sobrevivencia en el vivero. Lo cual se confirma con los resultados para esta especie empleando el sustrato b1 (con turba), el contenido de turba en este sustrato ayuda a mantener las plantas vivas en condiciones de vivero (ver Cuadro 4). Además de darle soltura a la mezcla, la turba proporciona nutrientes en tanto que retiene humedad (Borgo y Galloway 1987, Ocaña 1996). 
A los nueve meses del repique se observó que para B. tricuneata existe una mayor supervivencia $81-87 \%$ cuando se emplea el sustrato b1 (con turba), y de 70 - 87\% cuando se emplea b3 (sustrato simple). Sin embargo, el incremento de altura promedio resulto ligeramente mayor $1.04 \mathrm{~cm}$ empleando sustrato b3 y solo un incremento promedio de $0.9 \mathrm{~cm}$ empleado sustrato b1. Lo que indica poca diferencia entre estos dos sustratos para esta especie. Por otro lado, para esta especie no resulto viable el uso del sustrato b2 (sustrato arenoso) en el vivero, ya que se perdió toda la unidad experimental bajo este sustrato. Lo que confirma las conclusiones de Pérez Mercado (1994) con otras variedades de tola, que el flujo de agua del suelo a las hojas es mayor en Baccharis incarum Wedd. que en Parastrephia lepidophylla Wedd. determinando que en esta especie las pérdidas de agua son menores respecto a la especie $B$. incarum.

Alturas al final de la etapa de repique: Para $P$. quadrangularis los resultados del análisis estadístico arrojan diferencia significativa, con un coeficiente de variabilidad (CV) de $13.7 \%$ y altura promedio de $8.86 \mathrm{~cm}$ (ver Cuadro 6). La variabilidad en alturas (expresada en el valor del CV), sugiere que no existe diferencias cuando se trabajan las plantas sea cual sea los tamaños de bolsas (a1 y a2), pero sí hay diferencias cuando se trabajan las plantas en platabandas (a3), esto posiblemente se debe al área de suelo expuesto es mayor en platabandas lo que puede inducir a una acelerada pérdida de humedad en el suelo, sin embargo, los resultados podrían estar relacionados a la pérdida o la baja disponibilidad de minerales.

Para B. tricuneata, los resultados del análisis estadístico arrojaron que no existe interacción entre los factores, con un CV de 10,6\% y un promedio de altura total de $10.67 \mathrm{~cm}$. Este resultado es interesante porque la altura promedio de las plantas empleando los tres tratamientos varía entre $10.43-10.91 \mathrm{~cm}$; lo cual se puede tomar como un indicador de la plasticidad de la especie y posiblemente fácil manejo en condiciones de vivero, siempre y cuando no se utilicen sustratos arenosos con menos de 3.3 $\%$ de materia orgánica.
Perímetro al cuello de raíz al final del repique: Para $P$. quadrangularis el análisis estadístico indica que no existe significancia entre los factores $\mathrm{AB}$, y el CV $16.30 \%$ nos indica que los datos son regularmente variables; los factores no influencian el desarrollo del cuello de raíz utilizando a3 (platabandas) como técnica de propagación, a pesar de haberse realizado dos podas de raíz (al octavo mes y diez días antes de la plantación). Respecto a esto se sabe que, las especies nativas requieren una menor frecuencia de podas y recomiendan realizar la última poda de raíz 10-15 días antes de la plantación (PRONAMACHCS 1998). Sin embargo, para $B$. tricuneata el resultado del análisis estadístico, muestra que existe interacción entre los factores $\mathrm{AB}$, con un $\mathrm{CV}$ de $6.90 \%$ y perímetro promedio de $1.4 \mathrm{~cm}$. Observándose que la combinación alb3 (platabandas) tienen mayor desarrollo del cuello de la raíz $1.67 \mathrm{~cm}$. Esto se debe posiblemente a que las plantas trabajadas a raíz desnuda en la técnica a3, tiene un mejor desarrollo de la raíz en comparación con las plantas trabajadas en bolsas, lo cual se atribuyó a las podas de raíz; siendo la desventaja más saltante de esta técnica la producción de menores cantidades de plantas por área, que cuando se producen utilizando bolsas (Ocaña 1996).

Incremento mensual de las alturas: El desarrollo de ambos arbustos fue constante durante todos los meses que permanecieron en el vivero. Para $P$. quadrangularis desde abril hasta noviembre y para $B$. tricuneata desde mayo hasta noviembre; sin embargo, el incremento está influenciado por factores climático obteniéndose el menor desarrollo de la parte aérea $0.37 \mathrm{~cm}$ y $0.57 \mathrm{~cm}$ respectivamente en el mes de agosto con temperaturas media de 9.45 ${ }^{\circ} \mathrm{C}$; incrementándose el desarrollo de la parte aérea conforme la temperatura va incrementado y la precipitación se hace más frecuente.

\section{Etapa 4: Supervivencia en campo definitivo: plantación}

Para ambas especies el porcentaje de supervivencia fue alto por encima de $95 \%$ cuando las plantas fueron desarrolladas con los tratamientos a2, bolsa $13 \times 18 \mathrm{~cm}$, y a1, bolsa $10 \times 18 \mathrm{~cm}$, 
se debe posiblemente a la buena conformación radicular lograda mediante la poda y los cuidados durante el transporte, donde se controló y evitó exponer de forma directa las plantas al sol y el viento; también el uso del cepellón o pan de tierra apoyo a reducir el impacto negativo en las plantas durante la plantación, dependiendo esa reducción el grado de que se hayan disturbado las raíces. El éxito en el trasplante depende en gran parte del manejo previo de las plantas (Hartmann y Kester 1980). Por otro lado, se observó que las plantas propagadas a raíz desnuda técnica a3 platabandas, mostraron pérdidas por encima del $65 \%$ para ambas especies, esto probablemente se debe a un mayor impacto durante el trasplante, y quizás se requiera de un mayor tiempo en el vivero para poder obtener mejores resultados. Aunque, las plantas de $B$. tricuneata (a raíz desnuda) tuvieron una proporción ligeramente mayor en supervivencia en campo en comparación con $P$. quadrangularis, esto posiblemente a causa del mayor desarrollo de la raíz principal y raíces secundarias, que obtuvo $B$. tricuneata en platabandas. Por lo cual, $B$. tricuneata al parecer crece aceleradamente cuando se encuentra con superficie para desarrollar las raíces y las condiciones climáticas son favorables. Lo que puede estar conectado con las zonas donde se encuentra esta especie, por ejemplo, en el sur del país prospera en suelos pedregosos y de pendiente, exigiendo un mínimo de materia orgánica (Borel 1990). Lo cual puede atribuirse a esta especie como una ventaja una vez instalada en campo definitivo.

\section{Conclusiones}

La técnica de propagación empleando bolsas de $13 \times 18 \mathrm{~cm}$ obtuvo mejores resultados en vivero para $B$. tricuenata y $P$. quadrangularis, obteniendo más de un $80 \%$ de sobrevivencia al final del repique.

Se obtiene un mayor perímetro al cuello de raíz para $B$. tricuenata empleado platabandas y sustrato b1 (materia orgánica: tierra agrícola: turba). El incremento promedio de altura mensual para $B$. tricuneata fue de $1.04 \mathrm{~cm}$ con sustrato b1 (materia orgánica: tierra agrícola: turba). Mientras que para $P$. quadrangularis fue de $0.97 \mathrm{~cm}$ empleado el sustrato b3 (materia orgánica: tierra agrícola).

Empleando bolsas para ambas especies, la supervivencia en campo definitivo fue alta con más de $95 \%$ al mes de la plantación. Por otro lado, plantas producidas con la técnica platabandas a3 obtuvieron $27 \%$ y $31 \%$ de supervivencia para $P$. quadrangularis y $B$. tricuneata, respectivamente.

\section{Agradecimientos}

A la comunidad de San José de Aymará por el apoyo y permitir el estudio en las tierras de la comunidad.

\section{Referencias}

Borel, R. 1990. Especies agrosilvopastoriles para la zona altoandina. Pomata, Perú, Arbolandino. $205 \mathrm{p}$.

Borgo, G; Galloway, G. 1987. Manual de viveros en la sierra peruana. Lima, Perú, FAO. 123 p. Proyecto FAO Holanda/INFOR.

Brack, A. 1986. Ecología de un país complejo. En Gran Geografía del Perú. Vol. II. Edit. Manfer Juan Mejía Baca. Barcelona - España.

Brako, L; Zaruchi, J. 1993. Catalogue of the flowering plants and gymnosperms of Peru. Missouri, Estados Unidos de América, Botanical Garden. 1286 p.

Cabrera, AL. 1978. Flora de la provincia de Jujuy. Buenos Aires, Argentina. T. 13, pt. 10, 726 p. (Colección científica del INTA).

Dueñas, A. 2001. Efecto de fitorreguladores para el enraizamiento de diferentes especies de thola en la comunidad de Lleque Lleque, Santa Lucía. Puno, Perú, IIPSC QOLLASUYO. 4 p.

Hartmann, HT; Kester, DE. 1980. Propagación de plantas: principios y prácticas. México DF, México, Editorial Continental. 760 p.

IIPSC QOLLASUYO (Instituto de Investigación, Producción, Servicios y Capacitación Qollasuyo, Perú).2001.Producción e instalación de plantones de thola. Boletín técnico ${ }^{\circ} 2.8 \mathrm{p}$. 
Ocaña, D. 1996. Desarrollo forestal campesino en la región andina del Perú. Lima, Perú, FAO. 211p. Proyecto FAO/Holanda/ PRONAMACHCS/Perú.

PRONAMACHCS (Proyecto Nacional de Manejo de Cuencas Hidrográficas y Conservación de Suelos, Perú). 1998. Manual de producción de plantas forestales a raíz desnuda en la sierra peruana. Cajamarca, Perú. 137 p. Proyecto "Forestería en microcuencas altoandinas".

Pérez Mercado, R. 1994. Comportamiento hídrico-fisiológico y Fases fenológicas de la Thola. Tesis Ing. Agr. Oruro, Bolivia. 130 p.

Martínez, E. 2002. Caracterización morfológica y fitosociológica de las especies de thola en el ámbito de la cuenca Titicaca. Puno, Perú, IIPSC QOLLASUYO.

Reynel, C; Felipe-Morales, C. 1987. Agroforestería tradicional en los Andes del Perú: un inventario de tecnología y especies para la integración de la vegetación leñosa a la agricultura. Lima, Perú, FAO. 154 p. Proyecto FAO - Holanda/INFOR.

Reynel, C. 1988. Plantas para leña en el sur-occidente de Puno. Puno, Perú, MINAGRI. 165 p. Proyecto Arbolandino-Puno.

SENAMHI (Servicio Nacional de Meteorología e Hidrología del Perú). 2005. Parámetros climáticos de la Estación Acostambo a 3650 m.s.n.m.

Turner, BL; Kasperson, RE; Matson, PA; McCarthy, Jj; Corell, RW; Christensen, L; Eckley, N; Kasperson, JX; Luers, A; Martello, ML; Polsky, C; Pulsipher, A; Schiller, A. 2003. A framework for vulnerability analysis in sustainability science. Proceeding of the National Academy of Sciences 100(14):8074 - 8079.

Weberbauer, A. 1945. El mundo vegetal de los andes peruanos: estudio fitogeográfico. 2 ed. Lima, Perú, Ministerio de Agricultura. 776 p. 